\title{
APPROACH FOR THE REALIZATION OF UBIQUITOUS CONNECTIVITY IN ULTRA-DENSE WIRELESS ACCESS NETWORKS
}

\author{
Pavlina Koleva ${ }^{*}$ and Vladimir Poulkov \\ Technical University of Sofia, Faculty of Telecommunications, \\ 8, St. Kliment Ohridski Blvd, 1756 Sofia, Bulgaria, \\ e-mails:p_koleva@tu-sofia.bg,vkp@tu-sofia.bg
}

\begin{abstract}
This paper presents a novel user connectivity approach for future Ultra Dense Wireless Access Networks that will meet the requirements for user-centric ubiquitous access. The proposed technique will allow the implementation of such methods of utilization and management of the limited communication resources in the wireless access, through which an apparent to the user "unlimited capacity" will be provided, regardless of their type, number per unit area and mobility. The approach is based on an algorithm for user association, grouping and co-operative communication of Access Points, thus providing seamless, ubiquitous and instant connectivity and robust handover procedures.

Keywords: Access Networks, co-operative communication, heuristic optimization, Ultra-Dense Networks, user association.
\end{abstract}

\section{INTRODUCTION}

The rapid progress and the evolution of telecommunication technologies lead the world to a fully networked society where the access to communication resources and the exchange of information will be possible everywhere and at any time by anyone or everything. Today's vision for the future access network implies that access technologies will maintain connectivity between people, machines, devices, and a diverse range of everything else that can be connected. Even today, the huge increase of the multimedia traffic caused by the growing number of applications, social networking, online gaming, etc., along with the new types of communication technologies imposed by the Internet of Things (IoT) and Machine-to-Machine (M2M) communications, causes problems and challenges affecting the access to the network, the connectivity

${ }^{*}$ Corresponding author.

DOI: 10.7546/EngSci.LVII.20.01.02 
and the ability to transmit and process large volumes of data generated by end users and devices. In order to be able to address these challenges, to make possible the management of all these connected things and meet the increasing traffic requirements, the future generation of Access Networks (ANs) will have to introduce new functionalities and maintain a much wider range of services. Future ANs, especially those in Smart Cities (SCs), will also impose new requirements on the way the access to the network is realized, as they are expected to be very dynamic in nature and with a very dense user and Access Point (AP) distribution. In relation to all this, the issue of providing ubiquitous connectivity in such ultra-dense wireless ANs is very topical.

If we look at the development of the wireless ANs, it could be reasoned that at a certain stage in the development of mobile communications it will no longer be possible to increase the channel throughput due to Shannon's theoretically possible limits and bandwidth limitations. In these cases, the only possibility is to increase the bandwidth and/or to move to higher operating frequencies and higher density of placement of the Access Points (APs) [1]. The implementation of such ultra-dense deployments of different technologies and types of APs for ensuring higher data rates and ubiquitous information services will bring up issues with the resource management and provision of the required network QoS when transmitting uplink and downlink streaming multimedia traffic. In addition such complex and volatile mobility scenarios will raise complicated handover and load balancing issues $[2,3]$. The traffic load in SCs will fluctuate in space and over time due to the stochastic spatial distribution of users and APs in SC and user mobility [4]. For these challenges to be handled and for the realization of an effective access, new network architectures based on novel approaches for serving the users have to be proposed. In general, they have to implement novel user association schemes, based on grouping of active mobile terminals for cooperation, mobility prediction, and flexible solutions in coverage, i.e. to practically implement novel user association schemes and/or converged cell-less scenarios, as for example are the ones proposed in $[5,6]$.

This paper presents a novel user-centric connectivity approach for future Ultra-dense Access Networks (UdANs) that will meet the requirements for ubiquitous access and will allow the implementation of such methods of utilization and management of the limited communication resources in the wireless access, through which an apparent to the user "unlimited capacity" will be provided, regardless of their type, number per unit area and mobility. The paper introduces a method for association, grouping and co-operative communication of users and APs, thus providing seamless, ubiquitous and instant 
connectivity and robust handover procedures. The paper is organized as follows. In Section 2 the association approach is described, Section 3 illustrated the developed algorithm and some simulation results and the last Section concludes the paper.

\section{UTILITY FUNCTION AND USER ASSOCIATION APPROACH}

The implementation of dynamic association schemes for one or a group of users to one or more APs can be based on defining various utility functions for different system parameters. They can be considered static - depending on the wireless access infrastructure, or dynamic - related to the communication profile of users (human and machine) requesting service. Such utility functions can be used to achieve an Optimum Level of Performance (OPL) of the ANs that can be described as a target function in the following way:

$$
O P L=f u n c\left\{U E T_{i}, \sum_{j=1}^{k} U E A_{j}, U E L o c_{i}, M A X\left[u f_{i}\left(S T_{i}, p_{i}, P_{n}\right)\right]\right\}
$$

where $U E T_{i}$ refers to the type of user $i, \sum U E A_{j}$ to user activity, $U E L o c_{i}$ is the user location with respect to the available APs, $u f_{i}$ is a function of utility, required by user $i, S T_{i}$ is the type of service (voice, data, text, image, video), $p_{i}$ are the dynamic parameters such as the current channel state, user mobility, etc., and $P_{n}$ are static parameters depending on the specific AN infrastructure.

The proposed here connectivity approach gives a solution to the problem of dynamic connectivity management, on the one hand, based on new approaches and user association schemes and grouping of APs, and on the other on the basis of approaches for the implementation of cooperative work of groups of APs in order to ensure the necessary coverage and provide uninterrupted connectivity to users. The approach is based on proactively managing and dynamically connecting APs to a set of user requests for association and methods of APs grouping, and is implemented through an algorithm with low computational complexity that can provide seamless, ubiquitous, instantaneous and uninterrupted connectivity in UdAN.

The starting point for developing the dynamic association model is based on graph theory, and an UdAN is considered as a weighted non-directional graph. To implement the association model in real time, it is necessary to develop an algorithm combining the advantages of Heuristic Concentration, 
Linear Programming, and Graph Theory [7]. The model that is proposed is based on the two basic properties of APs: the attraction rate and the saturation rate [5]. In the model, the attraction rate is represented by a "Weight Matrix" using the graph theory and is a function of the coverage area of the AP. The weight of each arc is calculated in the same metric in which the attraction rate is measured. Thus, based on a "Weight Matrix", an assessment is made of the service (coverage) area boundaries of each AP. The saturation rate is represented by a "weight matrix of the graph nodes" describing UdAN. The weight of each node is a positive number corresponding to the saturation rate of the AP (in the appropriate metric of capacity, bandwidth or other applicable measure of the quantitative limit of the resources provided) or is zero if the node represents a user generating a request for association and service. Thus, the model defines two types of nodes - served (users) and serving (APs) represented respectively with a zero and non-zero value of the saturation rate. To form the "Weight Matrix", it is necessary to define an objective function that reflects the specifics of the UdAN operation as a connectivity provisioning system.

By using such a model of representation of the APs properties, it is possible to solve the following task: With a previously known user set and topology, as well as the nature and parameters of the association requests, an effective service system structure to be defined. In this regard, the AN architecture with the corresponding APs must be dynamically controlled in such a way that within each time slot the system is configured to ensure the condition of effective management of the AN, i.e. seamless and ubiquitous service of all user association requests and service provision with the required QoS through the optimal utilization of the resources of each AP. The key element of the dynamic association and grouping model is the definition of the objective function and the corresponding optimization task. The objective function is formed on the basis of three "price" components, one of which is static and two dynamic. The objective function reflects the "total cost" of associating and providing requested services and QoS to a given user $i$ to an $A P j$, at a distance $d_{i j}$ from user $i$ :

$$
\text { Objective Function }=P_{S}(i, j)+P_{O A M}(i, j)+P_{A P}(j),
$$

where $P_{S}(i, j)$ is the "price for service", $P_{O A M}(i, j)$ is the "price of OAM", $P_{A P}(i, j)$ is the "price of the APs". The first member of the objective function reflects the UdANs dynamics in terms of the resources that will be needed to provide user-requested services under a service scheme defined by the Association Matrix, i.e. which user from which AP will be served. The second 
and third members of the objective function present the "OAM price" of the $\mathrm{AP}$ and its associated infrastructure, depending on the user requests served as a result of the association. With such an approach the UdAN could be considered as a "cell-less" structure and one user in a given time slot can be associated with more than one AP, for example for the different directions of transmission or in case of cooperative service by several APs with the goal to provide the necessary capacity.

The purpose of the optimization task is to find the type of Association Matrix (matrix variable in the task) in which the value of the objective function reaches its minimum, satisfying certain conditions resulting from the limitations of the operation of UdANs as a service system without blocking. The optimization task consists in finding such a subset of $p$ active APs, $X_{P} \in X_{M}$ and $X_{P}=\{1, \ldots, p\}$, for which the generalized objective function (2) gets the minimum possible value under certain rules and constraints imposed by the Linear Programming, APs attraction rate, and APs saturation rate (capacity), taking into account the fact that APs need to serve user requests without buffering. Thus, it is necessary the generic objective function, reflecting the dynamic association of APs to the set of user service requests, to reflect also the dynamics within a serving time slot $\left(\Delta t_{s}\right)$ :

$$
\begin{aligned}
Z_{A P G}\left(\Delta t_{s}\right)= & \sum_{i=1}^{N\left(\Delta t_{s}\right)} \sum_{j \in X_{P}}^{p\left(\Delta t_{s}\right)} a_{i j}\left(\Delta t_{s}\right) w_{i j}\left(\Delta t_{s}\right) \\
& +\sum_{j \in X_{P}}^{p\left(\Delta t_{s}\right)} y_{j}\left[V s t a t_{j}+V d_{j}\left(\Delta t_{s}\right)\right] \\
= & \sum_{i=1}^{N\left(\Delta t_{s}\right)} \sum_{j \in X_{P}\left(\Delta t_{s}\right)}^{p} a_{i j}\left(\Delta t_{s}\right) w_{i j}\left(\Delta t_{s}\right) \\
& +\sum_{j \in X_{P}}^{p\left(\Delta t_{s}\right)} y_{j}\left[V \operatorname{stat}_{j}+f\left(\sum_{i=1}^{N\left(\Delta t_{s}\right)} a_{i j}\left(\Delta t_{s}\right) w_{i j}\left(\Delta t_{s}\right)\right)\right] .
\end{aligned}
$$

By applying the developed model and minimizing the generic objective function from expression (3), two key issues are solved related to the dynamics and configuration of UdANs as a service system with proactive management. The first is related to the determination of the number of active APs (medians) within the next slot, and the second to the creation a table (map) of user association requests for the next slot, i.e. which user to which active AP or Access Point Group (APG) is associated for service. Therefore, the optimization task is transformed into two-parametric and its formulation is related to 
finding the minimum of the objective function [8]:

$$
Z_{A P G}(p, A)=\sum_{i=1}^{N} \sum_{j=1}^{M} a_{i j} w_{i j}+\sum_{j=1}^{M} y_{j}\left(V s_{j}+V d_{j}\right)
$$

The specific feature of the objective function (4) is the second term that reflects the "price" of the active APs. The specific assessment of the "price" through the introduction of the dynamic component $V d_{j}$ is typical for an UdAN and reflects its functioning. It is assumed that the AP's "cost" within the current slot is a function of the served user requests for association without blocking.

\section{SIMULATION RESULTS}

For the purpose of the multi-parametric optimization of the objective function (4), it is necessary to develop an association and grouping algorithm that will allow simultaneous calculation of the value of $p$ (the number of active APs) and determination the type of Association Matrix $A$, in which $Z_{A P G}(p, A)$ reaches a minimum value. Given the dynamics of UdAN and the need for an efficient and with a relatively low computational complexity algorithm, the application of a heuristic algorithm for bi-parametrical optimization of the objective function (4), called in the sense of the applied heuristic procedure - ADD/DROP heuristics is proposed. The purpose of this heuristics is to define such an absolute median subset of APs in graph $G=\left(X_{N} \cup X_{M}, A\right)$ with dimension $p$ and members $X_{P} \in X_{M}$, where the objective function (4) reaches its absolute minimum value [9].

Figure 1 illustrates the block diagram of the ADD/DROP heuristic algorithm for dynamic user association and APs grouping by applying an ADD procedure.

The application of the ADD/DROP heuristic algorithm for user association and APs grouping for dynamic connectivity management in UdAN is illustrated through simulations. The user association and grouping of the APs according to the logic of the algorithm continues until such a median subset of active APs is obtained, whereby the objective function $Z_{A P G}(p, A)$ reaches its absolute minimum. The purpose of the simulation is to demonstrate that the APGs can be dynamically restructured taking into account the mobility of users maintaining connectivity and realizing the principle of "a network following the user" for the realization of ubiquitous connectivity. In the simulation scenario discussed here the parameters attraction rate $P a_{j}$ and saturation rate 


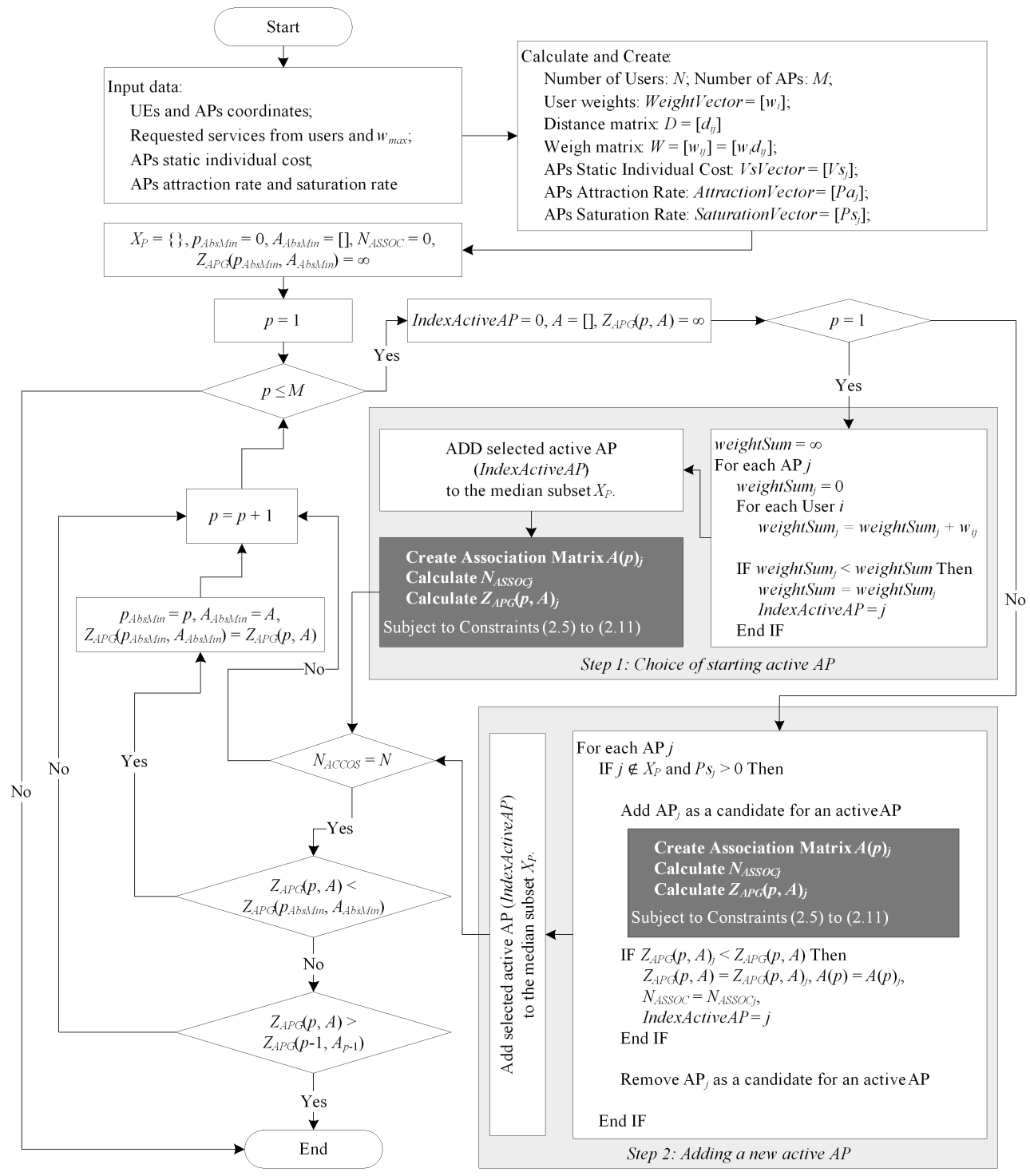

Fig. 1. Block diagram of the ADD/DROP heuristic algorithm for dynamic user association and APs grouping by applying ADD procedure

$P s_{j}$ are limited, each $\mathrm{AP} j$ can serve several users and it is allowed a given user $i$ to be associated for service to more than one active AP. The users are mobile and in the course of the simulation the user associations and the APGs are restructured in such a way to maintain constant connectivity and through the 


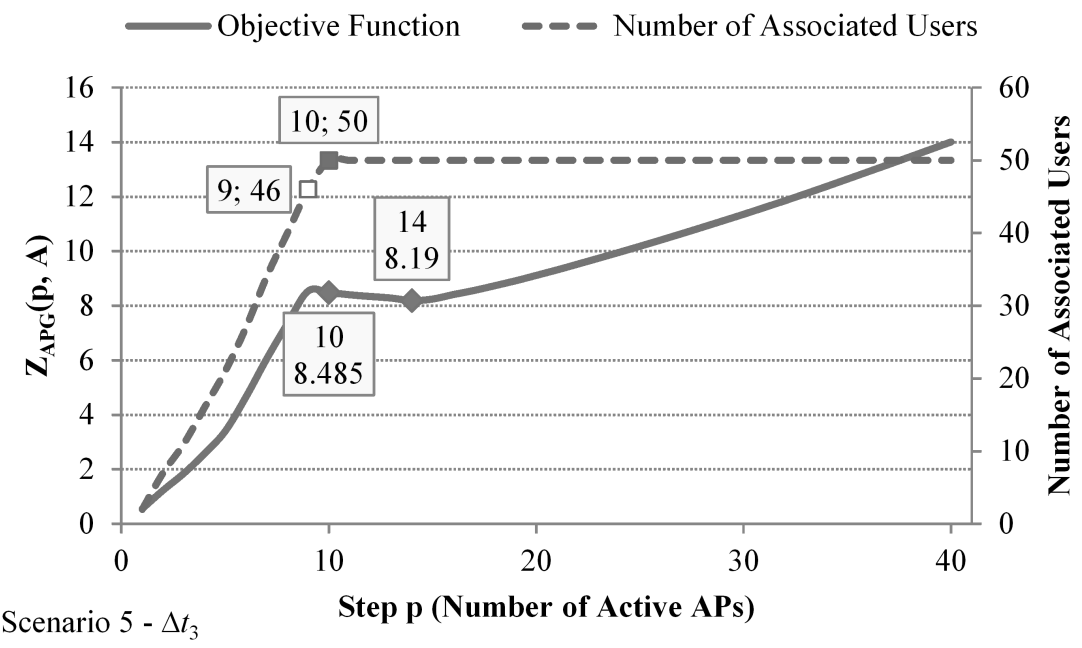

Fig. 2. Results of the calculation of the objective function for $\Delta t_{3}$

realization of the principle of "network following the user" to achieve ubiquitous connectivity.

In Figure 2 the results of applying the ADD/DROP heuristic algorithm for dynamic user association and AP grouping for one of the time slots of the performance of the algorithm are shown. It could be seen that after some steps of the algorithm the number of the associated users is constant (i.e. all users requesting connectivity are serviced) and further there is a point where the objective function reaches its minimum which means that the resources that are utilized for providing such ubiquitous connectivity are minimum.

The determination of the time slots or time periods for regrouping of the APs (or updating the APGs) depends on the structure and dynamics of the network, channel models, APs types and density, user mobility and density, etc., which could be reflected in the algorithm through appropriate determination of the attraction and saturation rates. Taking into account the dynamics of UdAN and that frequent regrouping will be required the heuristics and simple computational complexity of the proposed approach is the advantage for its application.

\section{CONCLUSION}

In this paper a model for proactive dynamic association of users and grouping of APs with low computational complexity based on the theory of graphs is proposed through the definition of an objective function for dynamic asso- 
ciation and grouping, based on static and dynamic components, and an optimization task for dynamic user association and AP grouping is formulated. To solve the optimization task, a heuristic algorithm for association and grouping has been proposed and developed, combining the advantages of Heuristic Concentration, Linear Programming and Graph Theory. The performance of the developed algorithm is illustrated by a simulation experiment for dynamically associating users for service to certain APs and/or APGs. The application of such an approach will allow a user to be associated and connected to a group of several cooperating APs to provide the requested throughput or QoS. The approach is analytical and is based on the capabilities of implementing a cooperative strategy to provide a higher probability of coverage of APs and the realization of optimal and ubiquitous connectivity of users through a dynamic organization of APG. The advantage of the proposed approach is that it uses criteria based on both the connectivity and coverage that each AP can provide and ensure, as well as its impact on the overall resource efficiency of the AN.

\section{ACKNOWLEDGEMENTS}

This work was supported by research project KP-06-N27/3/08.12.2018 "Resource self-configuration and management in ultra-dense networks with user centric wireless access" of the Bulgarian Scientific Research Fund.

\section{REFERENCES}

[1] Thomson Reuters, The World in 2025: 10 Predictions of Innovation, Technical Report (2014) (Online).

https://www.slideshare.net/sustainablebrands/the-world-in-2025-10-predictionsof-innovation

[2] A. Cimmino, T. Pecorella, R. Fantacci, F. Granelli, T. F. Rahman, C. Sacchi, C. CARlini, And P. Harsh, The role of small cell technology in future Smart City applications, John Wiley $\&$ Sons, Transactions on Emerging Telecommunications Technologies (2014) 25 (1) 11-20, DOI: 10.1002/ett.2766.

[3] F. Giust, L. Cominardi, And C. J. Bernardos, Distributed mobility management for future $5 \mathrm{G}$ networks: overview and analysis of existing approaches, IEEE Communications Magazine (2015) 53 (1) 142-149, DOI: 10.1109/MCOM.2015.7010527.

[4] X. Ge, J. Ye, Y. Yang, And Q. Li, User Mobility Evaluation for 5G Small Cell Networks Based on Individual Mobility Model, IEEE Journal on Selected Areas in Communications (2016) 34 (3) 528-541, DOI: 10.1109/JSAC.2016.2525439. 
[5] P. Koleva, V. Poulkov, and O. Asenov, Resource Management Based on Dynamic Users Association for Future Heterogeneous Telecommunication Access Infrastructures, Springer, Wireless Personal Communications (2014) 78 (3) 15951611, DOI: 10.1007/s11277-014-1911-z.

[6] T. Han, X. Ge, L. Wang, K. S. Kwak, Y. Han, and X. Liu, 5G Converged Cell-Less Communications in Smart Cities, IEEE Communications Magazine (2017) $\mathbf{5 5}$ (3) 44-50, DOI: 10.1109/MCOM.2017.1600256CM.

[7] M. J. Varnamkhasti, Overview of the Algorithms for Solving the P-Median Facility Location Problems, Hikari Journal, Advanced Studies in Biology (2012) 4 (2) 49-55.

[8] V. Poulkov, The Unified Wireless Smart Access for Smart Cities in the Context of a Cyber Physical System, in: Proceedings of Global Wireless Summit (GWS), Cape Town, South Africa, 15-18 October 2017, pp. 12-16, DOI: 10.1109/GWS.2017.8300499.

[9] J. E. Beasley, A note on solving large p-median problems, Elsevier, European Journal of Operational Research (1985) 21 (2) 270-273, DOI: 10.1016/03772217(85)90040-2.

Received March 31, 2020

Engineering Sciences, LVII, 2020, No. 1 